\title{
Interview with Ijad Madisch on "The Future of Publishing and Discussing Research"
}

\author{
Robert Winter
}

Published online: 7 March 2015

(C) Springer Fachmedien Wiesbaden 2015

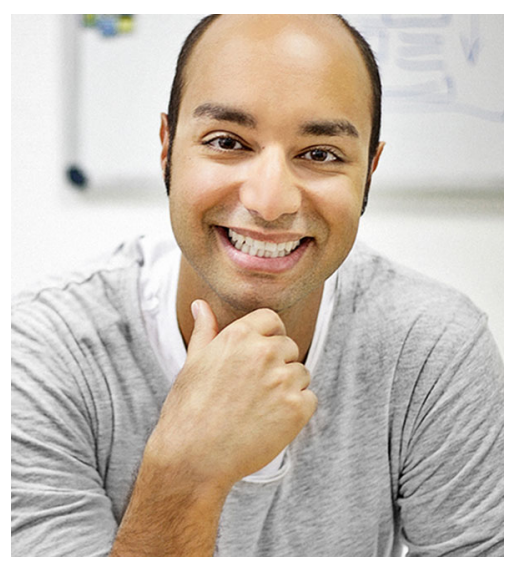

Dr. Ijad Madisch

ResearchGate $\mathrm{GmbH}$

Invalidenstr. 115

10115 Berlin

Germany

ijad.madisch@ researchgate.net

http://www.researchgate.net

Dr. Ijad Madisch is co-founder and CEO of ResearchGate, the professional network for scientists and researchers to collaborate, share results, and speed up progress. Following his own frustrations as a researcher isolated in his lab, Ijad Madisch founded ResearchGate in 2008 together with his friends, fellow physician Sören Hofmayer and computer scientist Horst Fickenscher.

Prof. Dr. R. Winter $(\square)$

Institute of Information Management, University of St. Gallen,

Mueller-Friedberg-Strasse 8, 9000 St. Gallen, Switzerland

e-mail: robert.winter@unisg.ch

URL: http://www.iwi.unisg.ch
ResearchGate has since grown to include more than six million scientists around the world. Ijad Madisch earned his doctorate in the field of virology, while also studying computer science on the side. He spent several years working as a researcher at Massachusetts General Hospital in Boston, focusing on tissue engineering and radiology. Under his leadership, ResearchGate has attracted a group of renowned investors, including Bill Gates, Benchmark and Founders Fund.

BISE: Do you see professional networks like ResearchGate as an addition or, in the long run, as a replacement for traditional forms for developing, publishing, and discussing research?

Madisch: Currently, it is a supplement. But we know from the past that an add-on can transform into a substitute. The journals need to show what their added value is. It used to be distribution and reputation that built up over time by quality assurance. The added-value of distribution has eroded because researchers can directly access results anytime and anywhere. Reputation is also becoming questionable because it does not relate to the research, but is instead inherited from the high impact of research previously published in that outlet. That has nothing directly to do with the publication. Regarding the third point, quality assurance, peer review has its own discussion. Researchers take over the reviewing task themselves, the publisher organizes it, and it has its advantages. Depending on the future developments of publishers' tasks, we will see whether they remain a part of the system, or if the system eventually transforms.

BISE: Now of course it is not your job to do the publishers' homework. How do you think will their role change?

Madisch: This is an extremely difficult question. I am glad that I do not have to think about it. The situation is 
similar to many other industries. If we take the automobile industry as an example, Mercedes has recently celebrated the installation of Apple software in its cars. I do not understand why Mercedes celebrates this. In my opinion that is the beginning of an end for Mercedes. Software is eating our world. Hardware is just a commodity. This device from Apple is not the best hardware that currently exists in the world. But the interaction between the software, hardware, and the data makes a system successful. I think in this regard the auto industry and the publishing industry are similar. Having information printed was important in a time when there was no Internet, no World Wide Web where information had to be transported. This is why journals existed. They have not developed further, in my opinion; they are still in the stagecoach time. I am glad that I do not have to be the one who has to consider what their task is in the future.

BISE: What insights can be gained from the huge collection of data in ResearchGate? What are you doing with the data, and what do you want to do?

Madisch: Well first of all, we do not sell data to third parties. There are ways to monetize data without selling data. Think about recruiting. We generate new data sets on job preferences that are the basis for a recommendationbased job board. It really creates high-quality applications within a short time for those who post a job there. We want to do the same for conferences. You will have the opportunity to advertise your conference to more relevant people who can contribute or want just to attend - maybe even from other disciplines. This is something we do well. The same applies to products and services. Imagine that you are looking for a statistician who will analyze large amounts of data, or a computer scientist who is not in your network, or someone in medicine or biology, or perhaps also a company that analyzes something for you. We want to create this market in a transparent way. The exciting fact is that we use already public data. When I read a publication, then I know what has been used and I can say, "If you want to buy that, then you go there." or "Send us your feedback about that product or service." Because we deal only with publications, with public data, it is a relatively simple story. We do not need any personal data to do this.

BISE: These are analyses or scenarios on an individual level. ResearchGate data might also create great potentials on the profile or network level. Is there already something planned?

Madisch: We have not done anything regarding that and we do not have any plan for that. This is something that we would rather leave to the academic institutions. Maybe in the future we might give data to academic institutions so they do the analyses. Currently, I cannot see so much to do there.
BISE: What about pattern recognition? Publications with promise to create high impact might be identified early by pattern matching, based on the impact data of existing publications.

Madisch: I had not thought about this yet and it is a good idea, to be honest. A new feature is also planned in this direction: simple recommendations for publications. What signals are included in this recommendation? This is a very, very difficult issue. Our best Russian and Ukrainian mathematicians are here to attack this problem. Let's start with co-author and institution networks. It is still relatively easy. But the next projects go exactly in this direction. The fact is that I got an idea while we have been here discussing this. This can be used somehow to present the right content to the right people. This is one of the features that we will focus on strongly in the coming months. We have millions of data uploaded every month, every day. People want to make their stuff visible. Now, the next step is to pass this information to the appropriate professors and researchers, because we believe that this is the right thing for them.

BISE: Although several public benefits might be created, ResearchGate is a start-up company, not a public institution. At the end how do you earn money?

Madisch: The types of investors we have do not necessarily want their money back. For example Bill Gates invested x-million from his 60 billion. I would like to call our project "responsible entrepreneurship." This is also reflected in our revenue streams. All revenue streams that we want to implement correlate positively with what we think researchers want. An example is recruiting. As a researcher, I was always interested in positions, not only in the academic world, but also outside of science. This is where I think we have a very unique position: to assign the right candidate to the right jobs and, vice versa, the right jobs to the right candidates. The second theme that I already mentioned is conferences. There is also a big "matching" problem because there are so many conferences out there and choosing the most appropriate one often is determined by personal experience or recommendations. The third theme is "Science as a service". In biology and medicine, this is already an established model. For example, it's easy for a statistician to do the statistics for a medical project. But when a physician tries that, half of the statistics may be wrong. This topic is more or less about outsourcing of scientific activities. There, I think, we can make money as well as provide useful services, without abusing the data.

BISE: What about growth limitations? In some research communities, ResearchGate participation is already more than $50 \%$. In business research it is much less. Nevertheless the growth cannot last forever. I can imagine participation might never move beyond $70 \%$ to $80 \%$. 
Madisch: Yes and no. We still have several areas where we want to grow. Some areas are missing, such as technicians. These are people who do not even think that they are researchers, but in fact they are! Take, for example, an MTA in the laboratory, who does not appear anywhere with his immense knowledge. The publications will always be authored by others. But what happens to all the technicians all over the world who actually have this very valuable practical knowledge? This is something where we can grow more strongly. Researchers outside of science are the second segment where we still want to grow. In the academic world we are doing well. There are some missing groups that we also want to attract, and then the whole nonacademic world.

BISE: But they have problems with the openness, right?

Madisch: Absolutely, the great challenge is to convince these companies that openness is an advantage for them. Big parts of Google and Facebook code are public. I call it Open Science. I believe that in science all can benefit when we work together more closely and more openly. It should not really matter whether I make a result public or not. Actually, I should try to make it public because I know that the contributions which I get through my contributions from the community have helped me to move forward. I think we need to change the consciousness of the corporate world in this direction.

BISE: Let us look now at the user's perspective: What about data maintenance? The most appropriate place to maintain the data would be the place where they are produced - with the author. All these platforms should offer an interface which allows authors to share - or not share their publication metadata. Today these metadata are dispersed across several platforms. With each new platform I need to upload my metadata. I can try to automate a certain part, or hope that my co-authors will do the job - but at the end I need to control all these metadata replicas. What is the vision of ResearchGate? Who manages the data and where? How open must such data, platforms or data management be?

Madisch: We have built features that address this issue, for example, by making it easy for a co-author to approve 'Yes, that paper belongs to that author'. We try to constantly improve this. Just recently a new version of ResearchGate has been released that learns from other social networks. Let's assume a network with ten people: if only one person among them is very active, the rest will automatically benefit. On the other hand, in the future we will want to create more functionality that replicates existing publication metadata, say from University sites or personal pages, back and forth. But this has to be well thought out, because an interface is not simple to build. By the collective intelligence of the networks, a lot of data can be uploaded. For example, each week around 10,000 conference papers would be uploaded.

BISE: Each system creates a potential for abuse. Where do you see the danger of abuse in ResearchGate, and what is your plan about it?

Madisch: This risk of abuse is always there, no matter what system you are using. This is somehow in our nature. What distinguishes us from all other networks aimed at the scientific community, is that people can register only with their institution email address. You cannot get ten email addresses on your institution account. This is a great added value for us. For example, the feature "Open Review" shows an abuse if all positive reviews come from the same institution - that is our strength. We can also tell people 'Your account will be locked' if they have been frequently reported to not comply with our standards - based on reports made by the community, not by us. That educates people. They cannot easily obtain another institutional account and start again.

BISE: Research (sub) communities differ in their publication usage patterns. E.g., while economists are journal oriented, computer scientists assign value also to conference proceedings. Some communities cite rather too much, others too little. Different community cultures should be reflected in the way their social network works - e.g., the way scores are calculated.

Madisch: We calculate scores at the segmented level. For example, for the business research community, let us assume the average number of citations is five times in that community. Researchers that are cited more than five times are considered to be above average in that community, while those cited less than five times are below average.

BISE: But if I have understood correctly, the way the score is calculated is the same for every research community. Should not different community cultures also lead to different score calculation schemes?

Madisch: Yes, definitely - that is still tough. We have a first draft. We optimize the scoring models regularly. The ultimate goal is to differentiate scoring models at the discipline level. That brings us closer to the truth in this discipline. For example, mathematicians or physicists do not publish often, but when they do it is giant papers with many authors. In contrast, social scientists make their working papers available online. While some put their project online before they start it, others only publish when it is ready.

BISE: Personal scores always trigger discussions and criticism. While you can claim that the ResearchGate score does not replace existing impact measures, the question is how much progress it creates and whether scientific measures develop in an appropriate direction.

Madisch: I think that journal metrics at some point will lose their significance. We know how they developed and 
what they measure - it is not necessarily the quality of a certain publication. While it is clear that we need an alternative, I think there is no broad consensus about what we actually want to measure. At ResearchGate, we believe that such measurements should not be based only on publications, but also on the data that you upload and on the questions that you answer. So it is a compound score.

BISE: We spoke briefly about the publishers at the beginning. What are actually the rules, when may researchers publish full-text on ResearchGate?

Madisch: As mentioned earlier, a full-text is not so terribly important to us - but this is actually the main point of conflict with publishers. Each journal has its own rules. There are the open-access journals where you have to pay a pretty large sum to get published. However, you do not really know what will happen at the end of the day with the publication. Then there are of course the traditional journals that have different regulations. Some allow you to publish the paper after 6 months. At the end of the day, ResearchGate is not about the publications; it is about the fact that we connect the right people, eventually leading to the request "Please, send me the paper". On a personal level, sending a paper should always be legal.

BISE: Is the posting of a non-formatted manuscript on ResearchGate considered as self-archiving?

Madisch: That is a grey area. In each country it is a little different. It is really crazy that we need to have this discussion at all and that we cannot do what we want with our work. At the end it is our intellectual creation. I hope that researchers begin to draw their conclusions and decide not to publish in certain outlets.

BISE: "It should be more important what we publish than where we publish". If (more or less) everything is published, filtering becomes key. How can a research platform support this increasingly critical task?

Madisch: That's correct. Today I can only say that it is definitely planned to better support intelligent filtering.

BISE: Dr. Madisch, thank you very much for this interview. 